\title{
Police Attitudes Toward Seeking Professional Mental Health Treatment
}

\author{
Jeanine Lane ${ }^{1} \cdot$ Marina Le ${ }^{1} \cdot$ Krystle Martin $^{1,2} \mathbb{D} \cdot$ Korri Bickle $^{1} \cdot$ Erin Campbell $^{1} \cdot$ Rosemary Ricciardelli $^{1,3}$
}

Accepted: 2 July 2021 / Published online: 15 July 2021

(c) The Author(s) 2021

\begin{abstract}
Public safety personnel (PSP), including police officers, often work in high-stress environments that expose them to potentially psychologically traumatic events (PPTE). As a result, PSP are at a higher risk than most other occupational roles for the development of new or worsening mental health concerns, such as posttraumatic stress disorder (PTSD), major depressive disorder, general or social anxiety disorder, and substance use disorders (Carleton et al., Canadian Journal of Psychiatry 63(1):54-64, 2018; Haugen et al., Journal of Psychiatric Research 94:218-229, 2017; Velazquez and Hernandez, Policing: An International Journal 42(4):711-724, 2019). Given these higher rates, research examining how to support the mental health of individuals in these occupations and how to improve our understanding of mental health help-seeking beliefs is critical. Consequently, the overall objective of the current study was to examine predictors of help-seeking attitudes among a group of police officers, while accounting for the effects of gender, years of employment, type of training experience, and mental health status (i.e. presence of mental illness, perceived resilience) on mental health-related help-seeking behaviours. A total of 112 police officers in a mid-sized law enforcement organization in Ontario, Canada, completed an online survey as part of a larger study exploring their mental health. Results indicate that years of experience and mental health training may improve attitudes toward seeking help for mental health. Our discussion offers suggestions for law enforcement organizations to consider to encourage their members to seek help for mental health difficulties.
\end{abstract}

Keywords Police officers $\cdot$ Public safety personnel $\cdot$ Mental health $\cdot$ Help-seeking $\cdot$ Stigma

\section{Introduction}

Public safety personnel (PSP), including police officers, often work in high stress environments with chronic and/ or extreme direct and indirect exposure to aversive experiences including violence, severe danger or human suffering, and tragedy (Bartol and Bartol 2008; Karaffa and Koch 2016; Kirschman et al. 2013). As a result of their occupation and exposure to potentially psychologically traumatizing events (PPTE), PSP are at a higher risk than other

Krystle Martin

martink@ontarioshores.ca

1 Ontario Shores Centre for Mental Health Sciences, 700 Gordon Street, Whitby, ON L1N 5S9, Canada

2 Faculties of Health Sciences and Social Science and Humanities, University of Ontario Institute of Technology, Oshawa, Canada

3 Department of Sociology, Faculty of Humanities and Social Sciences, Memorial University of Newfoundland, St. John's, Canada occupational groups and the general public for the development of new or worsening mental health concerns, such as posttraumatic stress disorder (PTSD), major depressive disorder, general or social anxiety disorder, and substance use disorders (Carleton et al. 2018; Haugen et al. 2017; Velazquez and Hernandez 2019). For example, researchers estimate $10-35 \%$ of PSP will develop PTSD over the course of their lifetime (e.g. Berger et al. 2012; DiGrande et al. 2011; Hartley et al. 2013; Maia et al. 2007; Oliphant 2016), compared to $9 \%$ of the general Canadian population (Kessler et al. 1995; van Ameringen et al. 2008). Given the higher prevalence of symptoms of mental disorders among PSP, research examining how to support the mental health of individuals in these occupations is essential. Accordingly, in the current study, we aimed to examine factors that predicted PSP's-specifically police officers-reported willingness to seek treatment for their mental health and attitudes toward mental health. 


\section{Seeking Treatment for Mental Health}

Although the World Health Organization (WHO) recommends evidence-based psychotherapies for the treatment of mood, anxiety, substance use, and trauma-related disorders, the majority of the general population with mental disorders delay seeking treatment (Wang et al. 2005). Delays in seeking treatment for mood disorders range from 6 to 8 years, and up to 9 to 23 years for anxiety disorders (Wang et al. 2005). As little as 7\% of people with PTSD in the general population will actually seek mental health services to receive treatment following trauma exposure, with the median time to seeking treatment being 12 years following symptom onset (Wang et al. 2005). Police officers are known to be even more reluctant than the general population to seek out mental health supports (Karaffa and Koch 2016; Karaffa and Tochkov 2013; White et al. 2016).

Many organizations have implemented programs to support the mental well-being of PSP; however, little evidence supports the effectiveness of these programs due to limited research (Oliphant, 2016). Furthermore, there remains a paucity of research on factors influencing police officers' help-seeking behaviours and attitudes toward mental health initiatives. This research is necessary to improve understanding of the barriers to accessing services, thus increasing the rate at which support is received.

Most researchers studying police officers' attitudes toward mental health have focused on officers' interactions with members of the public who present in psychiatric distress, whereas there is less literature on officers' attitudes toward themselves or colleagues with mental disorders. Some previous research has found officers' attitudes toward seeking professional mental health services to be neutral; however, officers also expressed concern regarding how to pragmatically utilize services, for instance not knowing where to access help (Karaffa and Tochkov 2013) or not having enough time (Martin et al. 2021). Other studies report ongoing stigma, especially for PSP who have mental disorders (e.g. Haugen et al. 2017). The culture within policing further presents as a challenge in discussing mental health with colleagues and managers, and therefore also in accessing support services (Bell and Eski 2016). A level of distrust of those outside the organization, in addition to officers not wanting to be perceived as weak for inquiring about mental health supports, further perpetuates reluctance in seeking out services (Karaffa and Tochkov 2013). Tailoring approaches to promoting and accessing services based on characteristics of the officers themselves is one strategy that may assist in encouraging more officers to seek therapeutic interventions. Currently, there is limited understanding of factors that predict an officer's attitude toward mental health and help-seeking behaviours.

\section{Gender and Sex}

Research indicates that men are overrepresented in policing (Mazowita and Greenland 2016), police officers' socialized gendered presentation is masculine (Miller et al. 2003; Silvestri 2017), and males in these roles are found to often stigmatize psychological services (Wester et al. 2010; Wester and Lyubelsky 2005). Police culture has historically been gendered involving values and traits traditionally characterized as masculine such as independence and emotional control, which may result in internalizing feelings-not expressing emotion - and not reaching out for social supports (Karaffa and Tochkov 2013). Thus, it has been suggested that gender and the gendering of the police officer profession may contribute to barriers to seeking treatment.

Scholars examining differences in male and female officers' attitudes toward, and help-seeking behaviours regarding, mental health have reported mixed results. For example, Montano and Barfield (2017), as well as Clayfield and colleagues (2011), indicated that female officers reported more positive attitudes toward mental health or persons with mental health concerns compared to male officers. Conversely, other studies did not find any differences in attitudes toward mental disorders between male and female officers (Cooper et al. 2004; White et al. 2016). However, these studies examined attitudes toward mental health more broadly, rather than the attitudes about the mental health of officers, either themselves or their colleagues.

Currently, any differences between male and female officers' attitudes toward mental health initiatives or toward the mental health of officers are not well established, and examining these differences in a relatively larger proportional sample of female officers is useful to unpack how gender or the gendered role of the officer informs attitudes toward help-seeking for mental health.

\section{Work Experience}

The number of years employed as an officer has not been found to be predictive of levels of stigma in an exploration of the link between years of service and attitudes toward mental health (Montano and Barfield 2017; White et al. 2016). Similar to the studies that explored gender differences, these studies examined attitudes toward mental health more generally, instead of officers' attitudes toward their own mental health or that of their colleagues. More research is needed to better understand if there are differences in attitudes and mental health stigma between officers with different levels of occupational tenure. 


\section{Impact of Training}

Corrigan et al. (2001) established that people with more knowledge about mental health have less negative attitudes toward individuals with mental disorders. For example, Hansson and Markstrom (2014) investigated the effectiveness of an antistigma intervention aimed at mental health in police officer training. Results indicated that, relative to the comparison group, the officers in the training group demonstrated improvements in their attitudes in terms of being open-minded and in intentional behaviour by indicating that they would be more willing to work with colleagues who have mental disorders. Clayfield et al. (2011) reported that officers with training related to responding to individuals in the community who present with mental health-related distress held more positive attitudes toward individuals in the community with mental disorders. Others have reported that training designed to address negative stereotypes in police officers or interventions related to mental health crises have led to better outcomes when dealing with both colleagues and members of the public with mental health needs (Cotton 2004; Watson and Fulambarker 2012). The literature indicates that providing mental health education for officers improves their competency in responding to members of the public with mental disorders, but as a result of improved attitudes toward mental disorders more generally, may also increase the likelihood of they themselves seeking services.

Montano and Barfield (2017), conversely, found the amount of specialized training for officers was not associated with their attitudes toward mental disorders. Limitedly, the authors did not qualify the type or intensity of the training received and also had a relatively small sample. Overall, the scope of mental health training necessary to change attitudes is unclear, and it is uncertain if additional training is helpful to decrease the stigma associated with mental health among police officers.

\section{Resilience}

Police officers' level of resilience may be relevant to their help-seeking behaviour. Research has indicated that resilience may be associated with fewer symptoms of mental disorders such as major depressive disorder and PTSD following PPTE exposure (e.g. Farr-Wharton et al. 2016; McCanlies et al. 2014; Ojedokun and Balogun 2015). A study examining fMRI results of officers exposed to the same traumatic event in three conditions (i.e. PTSD + officers provided psychotherapy; PTSD + officers on a waitlist; and symptom-free/resilient officers) provided neurophysiological evidence of resilience in a high-risk group of PSP (Peres et al. 2011). This study provides evidence not only for the importance of resilience in coping with the stress associated with being a PSP, but also for the benefit of accessing psychotherapy services in promoting improved resilience, shortening the period of suffering from symptoms and/or avoiding the chronicity of symptoms left untreated. However, research examining the effectiveness of training to improve resilience has reported limited outcomes and even negative attitudes toward the training (van der Meulen et al. 2018).

\section{Current Study}

Overall, there is a lack of literature examining predictors of officers' attitudes toward help-seeking behaviours for mental health despite the ongoing concerns that police are in special need of services to support them carrying out their duties and maintaining quality of life. Greater understanding of police officers' attitudes toward seeking mental health services will inform police services of factors to consider when developing and modifying training and wellness initiatives aimed to improve the mental health of officers, and subsequently, the health of the communities they serve.

The overall objective of the current study was to examine predictors of help-seeking attitudes among police officers, with a specific focus on gender, officer seniority, previous/ current mental health status, mental health training and selfreported resilience.

\section{Method}

\section{Study Design}

The current study used a quantitative design utilizing an online survey. It was part of a larger research project that focused on the creation of a mobile application resource aimed to support the well-being of police officers and civilian members of a mid-sized law enforcement organization (LEO) in Ontario, Canada, which was funded by the Ontario Ministry of Labour. ${ }^{1}$ Ethics approval was granted by the first author's institutional research ethics board and all data was collected in May and June of 2018.

\section{Participants and Recruitment}

All sworn staff from one Canadian municipal police service were eligible to participate in the study. Staff were notified via email from the LEO and provided with a link to an online

\footnotetext{
${ }^{1}$ Sponsored under the Ontario Ministry of Labour's Occupational Health, Safety, and Prevention Innovation Program, a new mental health App was created to support the mental health and wellness for members of the law enforcement agency included in this study. The data included in this paper was used to inform the design and content of this App.
} 
survey. Staff was informed that their participation was voluntary. Informed consent for participation was explained within the online invitation and implicitly received with engagement in the survey.

\section{Data Collection}

The online survey, which used Survey Monkey, had participants complete 35 questions about their demographics, employment, mental health, training and access to and use of mental health resources. In regard to demographic characteristics, participants were asked to report their gender (male, female, transgender, other, rather not say) and the year they were born. Employment items included the number of years they had been employed within a LEO and their rank. Participants also reported whether they had ever been diagnosed with a mental disorder (yes, no, prefer not to answer) and whether they were concerned about their mental well-being ("Are you currently concerned about your mental health? This might include your mood, experience of anger, level/frequency of anxiety, use of drugs or alcohol, etc."; yes, somewhat, no). The likelihood of seeking mental health treatment was examined using one question, which asked, "All in all, how likely is it that you would seek mental health treatment from a professional for a mental health concern you currently have or if you developed a mental health problem in the future?" to which participants were asked to rate by sliding an indicator along a line which produced a number between 0 and 100, with higher scores indicating greater likelihood.

There were two questions inquiring about participants' resilience: (1) "How resilient do you feel you are to manage your mental health?" which was rated by sliding an indicator along a line producing a number between 0 and 100, with higher scores indicating greater resilience; and (2) "How do you feel your resiliency to manage mental wellness has changed over time?" Participants were asked to indicate if their resiliency has increased, decreased, or stayed the same since they became a PSP.

Participants were also asked if they had ever participated in any training and/or education on the topic of mental health. Two courses that were specifically included were the Mental Health First Aid (MHFA) and the Mental Health Response Officer (MHRO) courses. The MHFA refers to a standardized 2-day workshop developed by the Mental Health Commission of Canada (MHCC) designed to teach attendees about "the help provided to a person developing a mental health problem, experiencing a mental health crisis, or a worsening of their mental health" (MHCC, 2020). The MHRO course is a 40-h program designed in-house by the LEO under study and is aimed at teaching police staff how to respond to and support individuals in the community who present with mental distress.

Final questions on the survey queried participants if they had ever participated in an event led by the critical incident stress management (CISM) team. They were asked to specify if they had attended a debrief-a structured intervention involving a discussion following a particularly distressing critical incident that occurs at work-or a defuse-which is a less formal, and shorter meeting after a critical incident that focuses more on providing psychoeducation to participants.

\section{Data Analysis}

Descriptive statistics were used to describe the sample and attitudes toward seeking mental health services. $T$-tests and an ANOVA were conducted to determine if there were differences in the likelihood of seeking treatment from a

Table 1 Participant characteristics

\begin{tabular}{ll}
\hline Group & $\begin{array}{l}\text { Sample size } \\
n(\%)\end{array}$ \\
\hline Gender & \\
Male & $88(78.6 \%)$ \\
Female & $23(20.5 \%)$ \\
Undisclosed & $1(1.0 \%)$ \\
Age $(M$, SD) & $44.58(7.3)$ \\
Years employed & \\
Less than 1 year & $2(1.8 \%)$ \\
1 to 3 years & $5(4.5 \%)$ \\
4 to 10 years & $8(7.1 \%)$ \\
11 to 20 years & $62(55.4 \%)$ \\
Over 20 years & $35(31.3 \%)$ \\
Mental health diagnosis & \\
No & $93(83.0 \%)$ \\
Yes & $19(17.0 \%)$ \\
Resilience $(M$, SD) & $80.42(18.3)$ \\
Has increased & $54(48.2 \%)$ \\
Has stayed the same & $31(27.7 \%)$ \\
Has decreased & $27(24.1 \%)$ \\
Mental Health Response course & \\
No & $29(25.9 \%)$ \\
Yes & $83(74.1 \%)$ \\
Mental Health First Aid & \\
No & $69(61.6 \%)$ \\
Yes & $43(38.4 \%)$ \\
No & \\
Yes & $89(79.5 \%)$ \\
Noitical incident-defuse & $23(20.5 \%)$ \\
\hline & \\
Yes & $37(33.0 \%)$ \\
\hline
\end{tabular}


mental health professional across different groups. Following, a multiple regression analysis to explore the predictive value of each of these variables with regard to help-seeking attitudes was conducted.

\section{Results}

\section{Participant Characteristics}

The current sample included 112 sworn police officers. As the participating LEO reported employing 904 sworn members, this represents an approximate participation rate of $12 \%$. The average age of participants was 44.58 years $(S D=7.3)$ and the majority $(n=88,80 \%)$ were male (note that for the purposes of this analysis, we excluded the one individual who chose not to disclose their gender; see Table 1). Police officers who had been working in their current occupation for less than 11 years were categorized as "junior officers" $(n=14 ; 13 \%)$ and those who had been working for over 11 years were categorized as "senior officers" $(n=96 ; 87 \%)$. Less than a quarter of participants self-reported having ever been diagnosed with a mental disorder $(n=19,17 \%)$. The participants reported a moderately high level of resilience with a score of 80.4 out of $100(\mathrm{SD}=18.3)$ and almost half $(48.8 \%)$ noted that their resilience has increased over time since becoming a PSP. Approximately one-third of participants had completed the LEO-designed course dedicated to supporting their professional development toward responding to individuals in the community who present with mental distress (MHRO), and about one-fifth of the sample reported completing the MHCC's mental health first aid course. While three-quarters of the sample acknowledged participation in a CISM debrief event, only one-third reported attending the less formal defuse format.

\section{Predictors of Mental Health Help-seeking Attitudes}

Overall, participants reported a fairly high likelihood they would seek mental health treatment from a professional with a mean of $84.7(\mathrm{SD}=20.46)$ out of 100 . Using a $t$-test, results indicated there were no significant differences between male and female officers in the likelihood of seeking treatment from a mental health professional. Similarly, using the Pearson $r$ correlation coefficient, neither self-reported level of resilience nor age was associated with likelihood to seek mental health treatment. However, there was a significant difference between officers who selfreported having a diagnosis of a mental disorder (now or in the past $)(t(110)=-2.61, p=0.01)$, indicating that officers who self-reported a diagnosis were more likely to indicate that they would seek mental health support. There were also significant differences between senior and junior officers in that responses of senior officers indicated that they are more willing to seek treatment from a mental health professional $(t(110)=-2.21, p=0.03)$ than junior officers. In addition, there were no reported differences between officers within the senior group (11 to 20 years compared to $>20$ years).

With regard to specific mental health training, officers who had completed the MHRO or the MHFA courses were more likely to indicate willingness to seek help for a mental health concern $(t(110)=2.43, p=0.02$ and $t(110)=2.94$, $p=0.00$, respectively) than those who did not complete a course. Further, a one-way ANOVA indicated that those who took both the MHRO and the MHFA courses indicated they were more willing to seek treatment for mental health than those who had taken neither of the courses $(F(3$, $27.86)=7.92, p=0.00)($ Table 3$)$.

There were no group differences between participants who had attended a CISM-related event (debrief or defuse) and those who had not on their likelihood of seeking mental health support from a professional.
Table 2 Factors that predict likelihood of seeking professional mental health support

\begin{tabular}{|c|c|c|c|c|c|c|}
\hline \multicolumn{2}{|c|}{ Model } & \multicolumn{2}{|c|}{$\begin{array}{l}\text { Unstandardized coef- } \\
\text { ficients }\end{array}$} & \multirow{2}{*}{$\begin{array}{l}\text { Standardized } \\
\text { coefficients } \\
\text { Beta }\end{array}$} & \multirow[t]{2}{*}{$t$} & \multirow[t]{2}{*}{ Sig } \\
\hline & & $B$ & Std. error & & & \\
\hline \multirow[t]{10}{*}{1} & (Constant) & 58.20 & 21.28 & & 2.74 & 0.01 \\
\hline & Gender (M/F) & 4.63 & 4.87 & 0.10 & 0.95 & 0.35 \\
\hline & Age & -0.36 & 0.32 & -0.13 & -1.13 & 0.27 \\
\hline & Mental health diagnosis $(\mathrm{Y} / \mathrm{N})$ & 3.96 & 2.40 & 0.18 & 1.65 & 0.10 \\
\hline & CISM debrief (Y/N) & 2.95 & 4.72 & 0.07 & 0.63 & 0.53 \\
\hline & CISM defuse (Y/N) & 3.91 & 4.34 & 0.10 & 0.90 & 0.37 \\
\hline & MHRO course & -2.55 & 4.20 & -0.07 & -0.61 & 0.55 \\
\hline & MHFA course & -8.37 & 4.86 & -0.19 & -1.72 & 0.09 \\
\hline & Seniority $(\mathrm{Jr} / \mathrm{Sr})$ & 27.75 & 7.29 & 0.42 & 3.81 & 0.00 \\
\hline & Resiliency (more/less) & -8.78 & 4.04 & -0.23 & -2.18 & 0.03 \\
\hline
\end{tabular}


Table 3 ANOVA: group differences-mental health training

\begin{tabular}{lllllll}
\hline Variable & & Sum of squares & df & Mean square & $F$ & $p$ \\
\hline Mental health training & Between groups & 4436.34 & 3 & 1478.78 & 3.80 & $0.01^{*}$ \\
& Within groups & $42,019.35$ & 108 & 389.07 & & \\
& Total & $46,455.68$ & 111 & & & \\
\hline
\end{tabular}

${ }^{*} p<0.05$
Multiple regression analysis was used to test what variables significantly predicted participants' ratings of likelihood of seeking professional mental health treatment (see Table 2). The results of the regression indicated the predictors explained $34.0 \%$ of the variance $\left(R^{2}=0.34, F(9\right.$, $71)=4.06, p=0.00$ ) (Table 3 ). It was found that seniority significantly predicted treatment seeking $(\beta=0.42, p=0.00)$, as did change in resilience $(\beta=-0.23, p=0.03)$. Casewise diagnostics revealed two outliers in the data set (participants with help-seeking scores more than 3 standard deviations from the mean). These outliers were removed and the regression was re-done (Table 4). With no outliers, the regression indicated that the predictors explained $33.0 \%$ of the variance $\left(R^{2}=0.33, F(9,69)=3.80, p=0.00\right)$. It was found that having been diagnosed with a mental health disorder predicted treatment seeking ( $\beta=0.24, p=0.04)$, as did taking the Mental Health First Aid course $(\beta=-0.27, p=0.02)$, seniority $(\beta=0.37, p=0.00)$ and change in resilience $(\beta=-0.23$, $p=0.03)$.

\section{Discussion}

The goals of the current study were to examine variables that might influence mental health help-seeking intentions and predictors of help-seeking attitudes among a group of police officers. The majority of the current sample endorsed willingness to seek mental health services from a professional if needed. This finding is different than what is generally reported in the literature which suggests that many officers are hesitant about seeking professional help for their mental well-being for a variety of reasons including stigma and pluralistic ignorance (Karaffa and Koch 2016) or that officers rely on other avenues to get support (i.e. social support and connection; Haas 2015). The difference could rest on that we asked our participants about willingness to seek help rather than measuring actual help-seeking behaviour.

When comparing groups, our findings also suggest that there are differences in the likelihood for help-seeking behaviour between police officers with self-reported mental disorders and between individuals who have completed certain mental health training workshops, despite the fact that these workshops were not about their own wellness but rather addressing the mental health needs of the community members they serve. However, when examining what predicted likelihood of help-seeking behaviour (after removing outliers), the results of the current study indicate that having been diagnosed with a mental health disorder, taking the Mental Health First Aid course, seniority and self-assessed change in resilience over time were significant predictors. This finding appears to counter stereotypes that younger generations hold fewer stigmatizing attitudes toward mental health and are more likely to seek services as compared to people from older generations (Bethune 2019). However, there may be other possible explanations as to why senior officers were more willing to seek professional help for mental health challenges. First, past research has shown that officers with more experience rate the importance of mental
Table 4 Factors that predict likelihood of seeking professional mental health support (outliers removed)

\begin{tabular}{|c|c|c|c|c|c|c|}
\hline \multicolumn{2}{|c|}{ Model } & \multicolumn{2}{|c|}{$\begin{array}{l}\text { Unstandardized coef- } \\
\text { ficients }\end{array}$} & \multirow{2}{*}{$\begin{array}{l}\text { Standardized } \\
\text { coefficients } \\
\text { Beta }\end{array}$} & \multirow[t]{2}{*}{$t$} & \multirow[t]{2}{*}{ Sig } \\
\hline & & $B$ & Std. error & & & \\
\hline \multirow[t]{10}{*}{1} & (Constant) & 82.93 & 16.52 & & 5.02 & 0.00 \\
\hline & Gender (M/F) & 1.76 & 3.64 & 0.05 & 0.48 & 0.63 \\
\hline & Age & -0.43 & 0.24 & -0.22 & -1.82 & 0.07 \\
\hline & Mental health diagnosis $(\mathrm{Y} / \mathrm{N})$ & 3.83 & 1.79 & 0.24 & 2.14 & $0.04^{*}$ \\
\hline & CISM debrief (Y/N) & -0.72 & 3.56 & -0.02 & -0.20 & 0.84 \\
\hline & CISM defuse (Y/N) & 2.84 & 3.28 & 0.10 & 0.87 & 0.39 \\
\hline & MHRO course & 0.51 & 3.15 & 0.02 & 0.16 & 0.87 \\
\hline & MHFA course & -8.70 & 3.62 & -0.27 & -2.41 & $0.02 *$ \\
\hline & Seniority $(\mathrm{Jr} / \mathrm{Sr})$ & 19.04 & 5.70 & 0.37 & 3.34 & $0.00^{*}$ \\
\hline & Resiliency (more/less) & -6.67 & 3.96 & -0.23 & -2.18 & $0.03^{*}$ \\
\hline
\end{tabular}


health training significantly higher than officers with less experience (Vermette et al. 2005). Thus, more experienced officers may also recognize the importance of tending to their own mental health concerns. Also, officers with greater seniority are likely exposed to more PPTE than those who are newer to the profession. The increased willingness to seek mental health services may be associated with greater need as a result of prolonged exposure to aversive experiences. Senior officers may also have a greater perceived sense of job security and may be less concerned about any negative impact of seeking services on their career.

In contrast, gender was not found to be a significant predictor of officers' willingness to seek mental health services, which is consistent with previous research that found attitudes and levels of stigma toward mental disorders did not differ between male and female officers (Cooper et al. 2004; White et al. 2016).

The results also point to the participants' perceived change in resilience over time since becoming a police officer as another predictor of help-seeking. Perhaps those individuals who reported that their resilience has decreased over time feel less able to engage in healthy activities or have less hope that professional support would be helpful, which could explain their reduced willingness.

Having been diagnosed with a mental health disorder was also found to be a significant predictor of willingness to seek help. Possibly, some of those who already had a diagnosis have reached out for help in past and this may lead to increased self-report scores on help-seeking. However, having been diagnosed does not mean that they have sought mental health support, something as routine as a visit to a family doctor can result in a mental health diagnosis.

Finally, having completed the Mental Health First Aid course was a significant predictor of willingness to seek help. Those who did not complete the course reported being less willing to seek help. It is possible that although the MHFA course is focused on the mental health of others, improving mental health literacy overall increases an individual's willingness to seek help and engage in activities to improve their own mental well-being.

Some limitations of the current study should be acknowledged and can inform future research. First, all outcome measures were based on the participants' self-report. As a result, outcome variables, such as resilience, were subject to officers' definition of the concept. Similarly, we only examined willingness-not behaviour-for help-seeking. This relates to the value-action gap observed and mostly described in social psychology (e.g. see Kollmuss and Agyeman 2002) whereby attitudes do not always correlate to actions. In our study, there may be a qualitatively critical difference between willingness and actually accessing mental health resources that is not captured in this research. As such, we recommend future qualitative work unpacking treatment-seeking behaviours among police officers. Additionally, our response rate was low (approximately 12\%) and so our sample size is relatively small. Also, it only includes staff from one LEO in Ontario, Canada. Given this, we note that our sample may not be representative of all Canadian police officers or LEOs. Moreover, we did not have equal number of officers in all groups, namely fewer female officers, and far fewer junior officers-future efforts should be made to recruit more participants from these groups in particular and to encourage more officers overall to respond to data collection efforts perhaps by offering incentives for participation.

\section{Implications and Conclusion}

The present study offers several insights to understand attitudes of help-seeking behaviour among Canadian police officers. There is research suggesting that police culture presents as a challenge in discussing mental health that may subsequently impact or create a barrier for staff to access support and services. Our intention in the current study was to first understand attitudes of officers more clearly to inform what type of support and change at the organizational/training level may be required. We hope that with specific highlevel changes, these will influence individual attitudes and possibly behaviour change when there is a supportive environment conducive to talking about, and accessing services for, mental health concerns. Considering, for example, the theory of planned behaviour, attitudes are one component of an individual's behavioural intentions (e.g. Ajzen 1991). Of course, we know that there are other factors involved in accessing mental health supports, like social norms (what do my colleagues and managers think about having mental health problems and asking for support/access/time off for seeking treatment), in addition to things like motivation and economic factors. The health belief model in part suggests that behaviour change is achieved by targeting and reducing barriers to change, as well as perceived benefits (Jones et al. 2015). Therefore, the more we can understand what influences beliefs about seeking help, the more specific and accurate our suggestions will be to organizations about mental health initiatives and supporting staff to seek mental health support.

As such, police services should direct attention toward factors that may pose as barriers for officers seeking help, such as shifting police culture to make engaging in mental health services normative and preferred. Given the finding that more junior officers were less likely to indicate willingness to seek mental health support, LEOs should direct extra attention to providing information and resources about mental health to new officers, for example while undergoing training and in early career follow-up supports. As it may be 
possible that junior officers are concerned about how accessing support would impact their career as has been reported in other research (e.g. Martin et al. 2021), it may be worthwhile to have senior officers who show willingness to seek professional support to deliver this information. This could be in the form of supervisors discussing mental health helpseeking and well-being during shift meetings, internal communication including personal stories from senior officers about their help-seeking attitudes, or use of force training officers (who are seen as officers in positions of authority) discussing this during annual training. Further, coach officers who provide $1-1$ training to new officers could incorporate discussions about wellness into their work with new recruits.

Previous research has found that more experienced officers rate the importance of mental health training significantly higher than officers with less experience (Vermette et al. 2005). Consistent with this, our finding demonstrates that officers who have attended workshops about mental health show more positive attitudes toward mental health help-seeking behaviour, which provides support for LEOs to offer continued education opportunities for their staff about mental health: even if it is not directed at their own wellness as this still seems to influence their personal experience. These results suggest the need for future research to ascertain the reasons for the shift in attitudes over time that officers described in our study to confirm our finding and better understand why senior officers report increasing willingness to access mental health support. Such information would be helpful as it could be used to inform efforts to address mental health and well-being with newer officers which has the potential to establish preventative efforts in support of long-term officer resilience.

In addition, LEOs should take care to monitor resilience among their staff. If feeling less resilient over time is indeed predictive of willingness to seek mental health support as our finding suggest, it would be critically important to share this information with officers and help them understand the consequences of this experience. These efforts have the potential to encourage officers to engage in regular self-care, check in with themselves on an ongoing basis and seek support if they notice a decline knowing that they will be at higher risk of refusing help if their resilience deteriorates. Our findings may also speak to the need for LEOs to make efforts to provide accurate information about the effectiveness of evidence-based psychotherapies and other mental health interventions to promote seeking help and provide hope.

Lastly, police officers endorsed a strong willingness to seek professional help for their mental health. This is very promising and should encourage LEOs to continue to educate staff by speaking about mental health and well-being in an open and direct manner. Attitudes about mental health help-seeking among police officers are perhaps shifting and any reticence on behalf of LEOs to encourage this behaviour may be unnecessary.

Funding This project was funded by the Ontario Ministry of Labour, Occupational Health, Safety and Prevention Innovation Program.

\section{Declarations}

Ethics Approval All procedures performed in this study involving human participants were in accordance with the ethical standards of the institutional and/or national research committee and with the 1964 Helsinki declaration and its later amendments or comparable ethical standards.

Informed Consent Informed consent was obtained from all individual participants included in the study.

Conflict of Interest The authors declare no competing interests.

Open Access This article is licensed under a Creative Commons Attribution 4.0 International License, which permits use, sharing, adaptation, distribution and reproduction in any medium or format, as long as you give appropriate credit to the original author(s) and the source, provide a link to the Creative Commons licence, and indicate if changes were made. The images or other third party material in this article are included in the article's Creative Commons licence, unless indicated otherwise in a credit line to the material. If material is not included in the article's Creative Commons licence and your intended use is not permitted by statutory regulation or exceeds the permitted use, you will need to obtain permission directly from the copyright holder. To view a copy of this licence, visit http://creativecommons.org/licenses/by/4.0/.

\section{References}

Ajzen I (1991) The theory of planned behavior. Organ Behav Hum Decis Process 50(2):179-211. https://doi.org/10.1016/07495978(91)90020-T

Bartol CR, Bartol AM (2008) Current perspectives in forensic psychology and criminal behavior. Sage Publications, Thousand Oaks CA

Bell S, Eski Y (2016) 'Break a Leg_-It's all in the mind': police officers' attitudes towards colleagues with mental health issues. Policing : A journal of policy and practice. [Online] 10 (2), 95-101

Berger W, Coutinho ESF, Figueira I, Marques-Portella C, Luz MP, Neylan TC, Marmar CR, Mendlowicz MV (2012) Rescuers at risk: a systematic review and meta-regression analysis of the worldwide current prevalence and correlates of PTSD in rescue workers. Soc Psychiatry Psychiatr Epidemiol 47(6):1001-1011

Bethune S (2019) Gen Z more likely to report mental health concerns. Monitor on Psychology, 50(1). http://www.apa.org/monitor/2019/ 01/gen-Z

Carleton RN, Afifi TO, Turner S, Taillieu T, Duranceau S, LeBouthillier DM, Sareen J, Ricciardelli R, MacPhee RS, Groll D, Hozempa K, Brunet A, Weekes JR, Griffiths CT, Abrams KJ, Jones NA, Beshai S, Cramm HA, Dobson KS, Hatcher S, Keane TM, Stewart SH, Asmundson G (2018) Mental disorder symptoms among public safety personnel in Canada. Can J Psychiatr [Online] 63 (1), 54-64 
Clayfield J, Fletcher K, Grudzinskas. Jr.A. (2011) Development and validation of the mental health attitude survey for police. Community Ment Health J 47(6):742-751

Cooper V, Mclearen A, Zapf P (2004) Dispositional decisions with the mentally ill: police perceptions and characteristics. Police Q 7(3):295-310

Corrigan PW, River LP, Lundin RK, Penn DL, Uphoff-Wasowski K, Campion J, Mathisen J, Gagnon C, Bergman M, Goldstein H, Kubiak MA (2001) Three strategies for changing attributions about severe mental illness. Schizophr Bull 27(2):187-195

Cotton D (2004) The attitudes of Canadian police officers toward the mentally ill. Int J Law Psychiatry 2(27):135-146

DiGrande L, Neria Y, Brackbill RM, Pulliam P, Galea S (2011) Longterm posttraumatic stress symptoms among 3,271 civilian survivors of the September 11, 2001, terrorist attacks on the World Trade Center. Am J Epidemiol 173(3):271-281

Farr-Wharton B, Azzopardi J, Brunetto Y, Farr-Wharton R, Herold N \& Shriberg A (2016) Comparing Malta and USA police officers' individual and organizational support on outcomes, Public Money \& Management, 36:5, 333-340. https://doi.org/10.1080/09540962. 2016.1194078

Haas NE, Van Craen M, Skogan WG, Fleitas DM. Explaining officer compliance: The importance of procedural justice and trust inside a police organization (2015) Criminology \& Criminal Justice, 15(4):442-463. https://doi.org/10.1177/1748895814566288

Hansson L, Markström U (2014) The effectiveness of an anti-stigma intervention in a basic police officer training programme: a controlled study. BMC Psychiatry 14(1):55

Hartley TA, Sarkisian K, Violanti JM, Andrew ME, Burchfiel CM (2013) PTSD symptoms among police officers: associations with frequency, recency, and types of traumatic events. Int J Emerg Ment Health 15(4):241

Haugen PT, McCrillis AM, Smid GE, Nijdam MJ (2017) Mental health stigma and barriers to mental health care for first responders: a systematic review and meta-analysis. J Psychiatr Res [Online] 94, 218-229

Jones CL, Jensen JD, Scherr CL, Brown NR, Christy K, Weaver J (2015) The Health Belief Model as an explanatory framework in communication research: exploring parallel, serial, and moderated mediation. Health Commun 30(6):566-576. https://doi.org/ $10.1080 / 10410236.2013 .873363$

Karaffa KM, Koch JM (2016) Stigma, pluralistic ignorance, and attitudes toward seeking mental health services among police officers. Crim Justice Behav [Online] 43 (6), 759-777

Karaffa KM, Tochkov K (2013) Attitudes toward seeking mental health treatment among law enforcement officers. Applied Psychology in Criminal Justice 9(2)

Kessler RC, Sonnega A, Bromet E, Hughes M, Nelson CB (1995) Posttraumatic stress disorder in the National Comorbidity Survey. Arch Gen Psychiatry 52(12):1048-1060

Kirschman E, Kamena M, Fay J (2013) Counseling cops: what clinicians need to know. Guilford Press, New York, NY

Kollmuss A, Agyeman J (2002) Mind the Gap: why do people act environmentally and what are the barriers to pro-environmental behavior? Environ Educ Res 8(3):239-260. https://doi.org/10. 1080/13504620220145401

Maia DB, Marmar CR, Metzler T, Nóbrega A, Berger W, Mendlowicz MV, Coutinho ES, Figueira I (2007) Post-traumatic stress symptoms in an elite unit of Brazilian police officers: prevalence and impact on psychosocial functioning and on physical and mental health. J Affect Disord 97(1-3):241-245

Martin K, Siddiqui A, Ricciardelli R, Lentz L, Carleton RN (2021) Differences in mental health, help-seeking, and barriers to care between civilians and sworn members working in law enforcement. J Police Crim Psychol (Online first). https://doi.org/10. 1007/s11896-021-09437-y
Mazowita B, Greenland J (2016) Police resources in Canada, 2015. Juristat: Canadian Centre for Justice Statistics, 1-22. Retrieved from https:// search.proquest.com/scholarly-journals/police-resources-canada2015/docview/1790516916/se-2?accountid=12378

McCanlies E, Mnatsakanova A, Andrew M, Burchfiel C, Violanti J (2014) Positive psychological factors are associated with lower PTSD symptoms among police officers: post Hurricane Katrina. Stress Health 30(5):405-415

Mental Health Commission of Canada (MHCC) 2020. (January, 2021). Mental Health First Aid Canada. https://mhfa.ca/

Miller SL, Forest KB, Jurik NC (2003) Diversity in blue: lesbian and gay police officers in a masculine occupation. Men Masculinities 5(4):355-385

Montano AN, Barfield B (2017) Attitudes toward mental illness: a study among law enforcement officers in the South and Southwest United States. Papers \& Publications: Interdisciplinary Journal of Undergraduate Research 6(1):11

Oliphant R (2016) Healthy Minds, Safe Communities: Supporting Our Public Safety Officers through a National Strategy for Operational Stress Injuries. Ottawa, ON, Canada:Standing Committee on Public Safety and National Security

Ojedokun O \& Balogun SK (2015) The costs of policing: Psychosocial capital and mental health outcomes in a Nigeria police sample. The Spanish Journal of Psychology, 18, Article E78

Peres JF, Foerster B, Santana LG, Fereira MD, Nasello AG, Savoia M, Moreira-Almeida A, Lederman H (2011) Police officers under attack: resilience implications of an fMRI study. J Psychiatr Res 45(6):727-734

Silvestri M (2017) Police culture and gender: revisiting the 'cult of masculinity'. Policing: A Journal of Policy and Practice 11(3), 289-300

Van Ameringen M, Mancini C, Patterson B, Boyle M (2008) Posttraumatic stress disorder in Canada. CNS Neurosci Ther 14(3):171-181

van der Meulen E, Bosmans M, Lens K, Lahlah E, van der Velden $P$ (2018) Effects of mental strength training for police officers: a three-wave quasi-experimental Study. J Police Crim Psychol 33(4):385-397

Velazquez E, Hernandez M (2019) Effects of police officer exposure to traumatic experiences and recognizing the stigma associated with police officer mental health. Policing: An International Journal. [Online] 42 (4), 711-724

Vermette HS, Pinals DA, Appelbaum PS (2005) Mental health training for law enforcement professionals. J Am Acad Psychiatry Law [Online] 33, $42-46$

Wang PS, Berglund P, Olfson M, Pincus HA, Wells KB, Kessler RC (2005) Failure and delay in initial treatment contact after first onset of mental disorders in the National Comorbidity Survey Replication. Arch Gen Psychiatry 62(6):603-613

Watson AC, Fulambarker AJ (2012) The crisis intervention team model of police response to mental health crises: a primer for mental health practitioners. Best Pract Ment Health 8(2):71

Wester SR, Arndt D, Sedivy S, Arndt L (2010) Male police officers and stigma associated with counseling: the role of anticipated risks, anticipated benefits and gender role conflict. Psychology of Men \& Masculinity 11(4):286-302

Wester SR, Lyubelsky J (2005) Supporting the thin blue line: gendersensitive therapy with male police officers. Prof Psychol Res Pract 36(1):51-58

White A, Shrader G, Chamberlain J (2016) Perceptions of law enforcement officers in seeking mental health treatment in a right-to-work state. J Police Crim Psychol 31(2):141-154

Publisher's Note Springer Nature remains neutral with regard to jurisdictional claims in published maps and institutional affiliations. 